\title{
Deep or partial caries removal: which is best?
}

\author{
In deep carious lesions, should all infected and affected dentine be removed \\ prior to restoration?
}

\author{
Thompson V, Craig RG, Curro FA, Green WS, Ship JA. \\ Treatment of deep carious lesions by complete excavation or partial \\ removal. A critical review. J Am Dent Assoc 2008; 139:705-712
}

Data sources Searches for studies were made using the databases: Medline, Evidence-based Medicine Reviews, Cochrane Database of Systematic Reviews, Cochrane Central Register of Controlled Trials and OVID's Database of Abstracts of Reviews of Effects.

Study selection Only studies reported in English with human participants were included. Randomised controlled trials (RCT) and relevant observational studies were included. No other inclusion or exclusion criteria are described.

Data extraction and synthesis A formal data extraction process is not described and a qualitative description of the included studies is provided.

Results Ten articles reporting six studies were included. The results of three RCT, one with a followup period of 10 years, provide evidence supporting the practice of leaving behind infected dentine, the removal of which would risk pulp exposure. A number of other studies show that cariogenic bacteria, once isolated from their source of nutrition by a restoration of sufficient integrity, either remain dormant or die and thus pose no risk to the health of the dentition.

Conclusions There is substantial evidence that, for caries management, it is not necessary to remove all vestiges of infected dentin from lesions approaching the pulp.
Address for correspondence: Dr R Craig, Department of Basic Sciences and Craniofacial Biology, New York University College of Dentistry, 345 East 24th Street/ 1001S, New York NY 10010-4086, USA. E-mail: rgc1@nyu.edu

\section{Commentary}

Since the days of GV Black, complete caries removal has been regarded as the gold standard in cavity preparation and, despite the lack of evidence to support this approach, it remains common practice today. In deep cavities, the carious process itself and the trauma of such radical caries removal can cause detrimental inflammatory changes within the pulp. If such caries removal leads to exposure of a vital pulp, a direct pulp cap, commonly using calcium hydroxide, is considered. Although most research on the direct pulp cap has focussed on the favourable outcome for traumatically exposed pulps which are healthy prior to the injurious incident, the prognosis following a carious exposure is not good: 5- and 10-year success rates of $37 \%$ and $13 \%$ respectively have been reported. ${ }^{1}$ This critical review is therefore appropriate, with its systematic search of the literature for evidence that might elucidate whether complete caries removal and its concomitant complications is necessary.

Carefully designed prospective RCT provide the strongest evidence for any intervention. Such trials were the focus of our 2006 Cochrane review $^{2}$ comparing complete or ultraconservative caries removal. The authors of this paper are correct, however, to draw attention to the fact that studies of a different design can also add to our understanding and can often provide compelling evidence for an intervention. Not including such studies may be regarded by some as "throwing the baby out with the bathwater". The aim here was therefore to extend the search and look for additional studies comparing complete or partial caries removal. In addition to two RCT included in the Cochrane review, four further studies were found, specifically three observational studies and one more RCT.

The main aim of the additional RCT was to investigate the cariostatic effect of black copper cement when partial caries removal was carried out in primary teeth. In the partial caries removal group, the durability of glass ionomer restorations lined with black copper cement was poor, but glass ionomer restorations alone performed as well following partial caries removal as they did following complete caries removal.

Of the three observational studies included here, two were stepwise excavation in all but name..$^{3,4}$ Although other similar clinical studies on stepwise excavation were not included in the main results of this paper, they were mentioned in the text (those cited in the Cochrane review $^{1}$ and the review by Bjørndal and Larsen, 2005). Three of these studies showed that caries that is left and sealed into the tooth after partial caries removal appears to arrest, so that when the cavities are re-entered the number of viable organisms within the lesions is significantly reduced. ${ }^{3-5}$

The final study included looked at the success of indirect pulp caps in primary molar teeth using either a calcium hydroxide lining material or resin-modified glass ionomer. The success rates presented at 4 years were $89 \%$ and $93 \%$, respectively. 
Although partial caries removal may sit uncomfortably with some dentists, the authors of this paper also describe a series of studies in which occlusal caries is arrested by simply fissure-sealing the lesions with no caries removal at all. Other studies are described that add further weight to the partial caries removal argument. These all show that by depriving the organisms within lesions of the intra-oral substrate they require to survive, both the number and diversity of organisms decline, with only those able to metabolise pulpal serum proteins surviving. ${ }^{6}$ These organisms are not associated with active carious lesions, and even pulpal nutrients will decline with time because of pulp-dentine complex reactions of tubular sclerosis and reactionary dentine formation.

Unfortunately, it is not clear from this review, or the original papers, what constitutes deep caries or partial caries removal. Some authors have described lesions reaching up to halfway to the pulp, determined on a radiograph, whereas others have given little specific information other than saying the lesion is deep, or adding that the extent means pulpal exposure is likely if caries is completely removed. Similarly, partial caries removal varies from simply bevelling enamel at the entrance to the fissure to carrying out only peripheral caries removal and leaving soft infected carious dentine pulpally; to removal of caries until firm, stained dentine is reached and then placement of an indirect pulp cap.

The studies cited are therefore heterogeneous, but the evidence stemming from them all is that removal of all carious tissue is not necessary. In light of the substantial evidence cited to support partial caries removal, the authors of this paper point out that there have, as yet been no studies to prove the desirability of removing all infected dentine. They conclude that, "before this concept (of partial removal) is generally accepted by the profession additional clinical trials may be needed". This, I am sure, is true. These trials should be carried out in primary care with detailed, specific information on lesion extent and what constitutes partial caries removal. The success of such interventions also needs to be assessed along with research into techniques for monitoring sealed caries.

\section{David Ricketts}

Department of Restorative Dental Care and Clinical Dental Sciences, University of Dundee Dental School, Dundee, Scotland, UK

1. Barthel CR, Rosenkranz B, Leuenberg A, Roulet JF. Pulp capping of carious exposures: treatment outcome after 5 and 10 years: a retrospective study. J Endod 2000; 26:525-528.

2. Ricketts DN, Kidd EA, Innes N, Clarkson J. Complete or ultraconservative removal of decayed tissue in unfilled teeth. Cochrane Database Syst Rev 2006; issue 3.

3. Maltz M, de Oliveira EF, Fontanella V, Bianchi R. A clinical, microbiologic, and radiographic study of deep caries lesions after incomplete caries removal. Quintessence Int 2002; 33:151-159.

4. Fairbourn DR, Charbeneau GT, Loesche WJ. Effect of improved Dycal and IRM on bacteria in deep carious lesions. J Am Dent Assoc 1980; 100:547-552.

5. Bjørndal L, Larsen T. Changes in the cultivable flora in deep carious lesions following a stepwise excavation procedure. Caries Res 2000; 34:502-508.

6. Paddick JS, Brailsford SR, Kidd EA, Beighton D. Phenotypic and genotypic selection of microbiota surviving under dental restorations. Appl Environ Microbiol 2005; $71: 2467-2472$.

Evidence-Based Dentistry (2008) 9, 71-72. doi:10.1038/sj.ebd.6400592 